\title{
Important role of long non-coding RNA UBE2R2-AS1 in diabetes-induced renal injury
}

\author{
Chuanqi Chen, Yanqiong Yan, Qianjun Luo, Sufen Li
}

Endocrinology Department, Shekou People's Hospital, Nanshan District, Shenzhen, Guangdong, China

Submitted: 26 January 2020

Accepted: 7 March 2020

Arch Med Sci

DOI: https://doi.org/10.5114/aoms.2020.100649

Copyright $\odot 2020$ Termedia \& Banach

\begin{abstract}
Introduction: The present study examined the effects and mechanisms underlying long non-coding RNA (InCRNA) UBE2R2-AS1 in diabetes-induced renal injury.

Material and methods: High glucose was used to imitate diabetes-induced renal injury in a cell model. Cell apoptosis rate was measured using flow cytometry, tumour necrosis factor- $\alpha$ (TNF- $\alpha$ ) and interleukin- 6 (IL-6) concentrations were evaluated using ELISA, and relative protein expression and amount were measured using western blot (WB) analysis and immunofluorescence, respectively. Correlations between IncRNA UBE2R2-AS1, miRNA-877-3p and TLR4 were analysed using the luciferase reporter assay.

Results: Cell apoptosis rate and TNF- $\alpha$ and IL- 6 concentrations were significantly higher $(p<0.001)$ in the high glucose (model) group compared with those of the Control group (CG) group. Following shRNA knockdown of IncRNA UBE2R2-AS1, the cell apoptosis rate and TNF- $\alpha$ and IL- 6 concentrations were significantly lower ( $p<0.001$, respectively) compared with those of the model group. However, under high-glucose conditions, shRNA knockdown of UBE2R2-AS1 and miRNA-877-3p significantly increased $(p<0.001)$ the cell apoptosis rate as well as TNF- $\alpha$ and IL- 6 concentrations compared with the shRNA UBE2R2-AS1 knockdown group. WB and immunofluorescence analysis showed that toll-like receptor 4 (TLR4)/myeloid differentiation factor 88 (MyD88)/nuclear factor $\kappa \mathrm{B}$ (p65) (NF- $\mathrm{kB}(\mathrm{p} 65)$ ) pathway proteins were significantly stimulated in the model group compared with those in the CG, whereas shRNA transfection with miRNA-877-3p stimulation suppressed the TLR4/MyD88/NF-кB(p65) pathway.

Conclusions: Knockdown of IncRNA UBE2R2-AS1 improves diabetes-induced renal injury via regulation of the miRNA-877-3p/TLR4 axis in vitro.
\end{abstract}

Key words: UBE2R2-AS1, renal injury, diabetes, miRNA-877.

\section{Introduction}

Diabetic kidney disease (DKD) is the most frequently occurring complication of diabetes mellitus, and it is the primary cause of end-stage renal disease and one of the main causes of death in patients with diabetes. Data from the 2015 IDF Diabetes Atlas [1] revealed that there were approximately 415 million patients with diabetes worldwide, accounting for 1 in every 11 adults. The number of patients with diabetes was predicted to reach 642 million by 2040. In China, there are presently 109 million patients with diabetes, and this number is expected to reach

\author{
Corresponding author: \\ Chuanqi Chen \\ Endocrinology \\ Department \\ Shekou People's \\ Hospital \\ Nanshan District \\ Shenzhen \\ Guangdong, China
}


151 million by 2040. DKD is a progressive disease and is the second-most common complication in the late stage of diabetes and a major cause of chronic renal failure, making it a major cause of morbidity and mortality among patients with diabetes [2]. The pathological features of DKD include mesangial expansion and hypertrophy, protein deposition in extracellular matrix (ECM) and podocyte apoptosis [3], followed by glomerular sclerosis, tubulointerstitial fibrosis and eventually renal failure.

Long non-coding RNAs (IncRNAs) are a class of RNA molecules that do not encode proteins and are typically more than 200 nucleotides in length. While recent studies have confirmed that some IncRNAs as important regulators involved in the development of diabetes [4-10], their precise role in DKD remains unclear. Overexpression of IncRNA UBE2R2-AS1 promotes tumour cell apoptosis; however, its role in high-glucose-induced renal tubular epithelial cell apoptosis has not yet been elucidated. In our present study, we discuss the effects and mechanisms of IncRNA UBE2R2-AS1 in renal injury induced by diabetes in an in vitro study.

\section{Material and methods}

\section{Materials}

The following chemicals and assay kits were used: DMEM F-12 medium (Hyclone, USA); foetal bovine serum (Gibco, USA); penicillin, streptomycin and amphotericin B (Lonza, USA); trypsin (GibCo, USA); TRIzol reagent (Invitrogen, USA); CDNA reverse transcription kit (Invitrogen, USA); SYBR green PCR fluorescent dye (Invitrogen, USA); human TNF- $\alpha$ and IL-6 ELISA Kit (R\&D, USA); mouse anti-rabbit TLR4, MyD88 and NF- $\kappa B$ (p65) antibodies (Abcam, UK); horseradish peroxidase (HRP) labelled anti-rabbit and anti-mouse secondary antibodies (Beijing Dingguo Changsheng); fluorescence labelled anti-mouse and anti-rabbit secondary antibody (Invitrogen, USA); flow cytometry kit (Jiangsu Keygen Biotech, China); shRNA-negative control (shRNA-NC) and IncRNA UBE2R2-AS1 inhibitor, shRNA-UBE2R2-AS1 and miR-887-3p inhibitor (Jiangsu Keygen Biotech, China).

\section{Cell culture}

The human proximal tubular epithelial cell line HK-2 was purchased from the American Standard Culture Collection and was cultured in DMEM-F12 medium containing 10\% fetal bovine serum, $104 \mathrm{U} /$ / penicillin, $104 \mathrm{U} / \mathrm{I}$ streptomycin and $20 \mathrm{mg} / \mathrm{l}$ amphotericin $\mathrm{B}$ in a humidified incubator at $37^{\circ} \mathrm{C}$ with $5 \% \mathrm{CO}_{2}$. Cells were observed under an inverted microscope and were observed to merge into a monolayer, displaying a typical paving stone-like arrangement after around 3 days.

\section{Cell grouping and stimulation}

Cells were seeded in 6-well plates and synchronised for $12 \mathrm{~h}$ in serum-free DMEM-F12 medium until they reached $70 \%-80 \%$ confluence. HK-2 cells were divided into three groups as follows: the CG was cultured in conventional DMEM-F12 medium, the model group was treated with high glucose (D-glucose $30 \mathrm{mmol} / \mathrm{l})$; the model + shRNA-NC group was transfected with non-targeting shRNA prior to treatment with high glucose; the model + shRNA group was transfected with shRNA targeting UBE2R2-AS1 prior to treatment with high glucose, and the model + shRNA + miRNA inhibitor group was co-transfected with shRNA-UBE2R2-AS1 and miR-877-3p inhibitor prior to treatment with high glucose. The cells were transfected with shRNA-NC, shRNA-UBE2R2-AS1 or miR-877-3p at room temperature for $12 \mathrm{~h}$.

\section{Flow cytometry analysis of apoptosis}

Apoptosis was induced in each group as previously described. Cells $\left(5 \times 10^{5}\right)$ were collected by centrifugation and resuspended in $1 \times$ binding buffer (diluted from $5 \times$ binding buffer with distilled water). Next, $5 \mu \mathrm{l}$ of FITC and $10 \mu \mathrm{l}$ of propidium iodide were added to each tube, gently mixed and incubated in the dark for 5 min prior to flow cytometry analysis.

\section{Measurement of inflammatory factor concentrations by ELISA}

Supernatants were collected from the cells from each group to measure the concentrations of inflammatory factors IL- 6 and TNF- $\alpha$, using ELISA kits according to the manufacturer's protocol (Sigma, USA).

\section{RT-PCR measurement of IncRNA UBE2R2-AS1 and miR-877-3p mRNA expression}

Total RNA was extracted from cells from the different groups using TRIzol reagent and the concentration determined using an ultraviolet spectrophotometer. cDNA was synthesised by reverse transcription using $2 \mu \mathrm{g}$ of RNA from each group in a $20-\mu \mathrm{l}$ reaction system, according to the manufacturer's instructions (Toyobo, Japan). Primers were synthesised from Jiangsu Keygen Biotech as follows: IncRNA UBE2R2-AS1 F: 5'-GTCTGGGTAGTCAGCTGTGAGG-3', R: 5'-TCTCCAGAGGCAGTGTTCCTC-3'; miR-877-3p F: 5'-TCCTCTTCTCCCTCCTCCC-3', R: 5'-CTCTACAGCTATATTGCCAGCC-3' and U6 F: 5'-GGGAGATACCATGATCACGAAGGT-3', R: 5'-CCACAAATTATGCAGTCGAGTTTCCC-3'. Pre-denaturation was performed at $95^{\circ} \mathrm{C}$ for $10 \mathrm{~min}$ followed by 40 cycles of denaturation at $95^{\circ} \mathrm{C}$ for $10 \mathrm{~s}$, annealing at $60^{\circ} \mathrm{C}$ for $20 \mathrm{~s}$ and extension at $72^{\circ} \mathrm{C}$ for $15 \mathrm{~s}$. The relative expression of the target genes was calculated using the $2^{-\Delta \Delta C T}$ 
method. Three duplicate wells were set up for each group, and the experiment was repeated three times in an in vitro study.

\section{Western blot analysis of protein expression}

Cells from each group were collected and lysed to extract total protein using lysate buffer, and protein concentration was measured using a BCA kit (Sigma, USA) according to the manufacturer's instructions. Total protein samples $(50 \mu \mathrm{g})$ were taken from each group, separated using 10\% SDS-PAGE, electrotransferred to PVDF membranes, blocked using $5 \%$ skimmed milk at room temperature for $2 \mathrm{~h}$, washed with PBST (phosphate buffered saline) and incubated with anti-TLR4, anti-MyD88, anti-NF- $\mathrm{B}$ (p65) or anti-GAPDH antibody (1: 1000 for all) overnight at $4^{\circ} \mathrm{C}$. The next day, after rinsing with TBST (Tris-Buffered Saline and Tween 20), HRP-labelled goat anti-rabbit and anti-mouse IgG was added and incubated for $2 \mathrm{~h}$ at room temperature. Bands were visualised using ECL reagents, using GAPDH as an internal control. The experiment was repeated three times under the same experimental conditions and the grey value of the target bands was determined using Image J software. This experiment was repeated 3 times

\section{Immunocytochemistry measurement of TLR4, MyD88 and NF-kB (p65) protein expression}

Cells were cultured in six-well plates, washed with $4 \%$ paraformaldehyde for $20 \mathrm{~min}$, permeabilised with $0.5 \%$ Triton X-100 for 30 min and incubated with a drop of primary antibody diluted with $1 \%$ bovine serum albumin (BSA) at $4^{\circ} \mathrm{C}$ overnight. The next day, secondary antibody (diluted 1:200 with $1 \%$ BSA) was added dropwise and incubated at $37^{\circ} \mathrm{C}$ in the dark for $1 \mathrm{~h}$. The slice was then washed with PBS and sealed with DFX-containing anti-quenching sealer and observed under a fluorescence microscope. Fluorescence images were analysed using Image-Pro Plus 6.0 software. Ten fields were randomly selected for each group and the average fluorescence intensity of each field was calculated by dividing the integral fluorescence intensity by the area of the field.

\section{Luciferase reporter assay}

Cells were transfected with empty vector or miR-877-3p using luciferase reporter containing 3'-UTRs of TLR4, wild-type or mutant UBE2R2-AS1 fragments using Lipofectamine 2000 (Invitrogen) in 96-well plates. Cells were harvested for $48 \mathrm{~h}$ after transfection and luciferase activity was measured using chemiluminescence in a luminometer (PerkinElmer Life Sciences) using the Dual Luciferase reporter assay system (Promega).

\section{Statistical analysis}

Relative data were analysed by SPSS 22.0 and graphs were drawn using GraphPad Prism 7.0. Data were expressed as mean \pm SD, $t$ test was used for pairwise comparison and one-way analysis of variance was used for comparison among multiple groups. $P$-values $<0.05$ were considered statistically significant. All experiments were repeated 3 times.

\section{Results}

\section{LncRNA UBE2R2-AS1 and miRNA-877-3p gene expression}

RT-PCR analysis revealed that UBE2R2-AS1 mRNA expression was significantly higher $(p<0.001)$ in the model and model + shRNA-NC groups, whereas miRNA-877-3p mRNA expression was significantly lower $(p<0.001)$ in these groups compared with the CG (Figure 1). Compared with the model group,
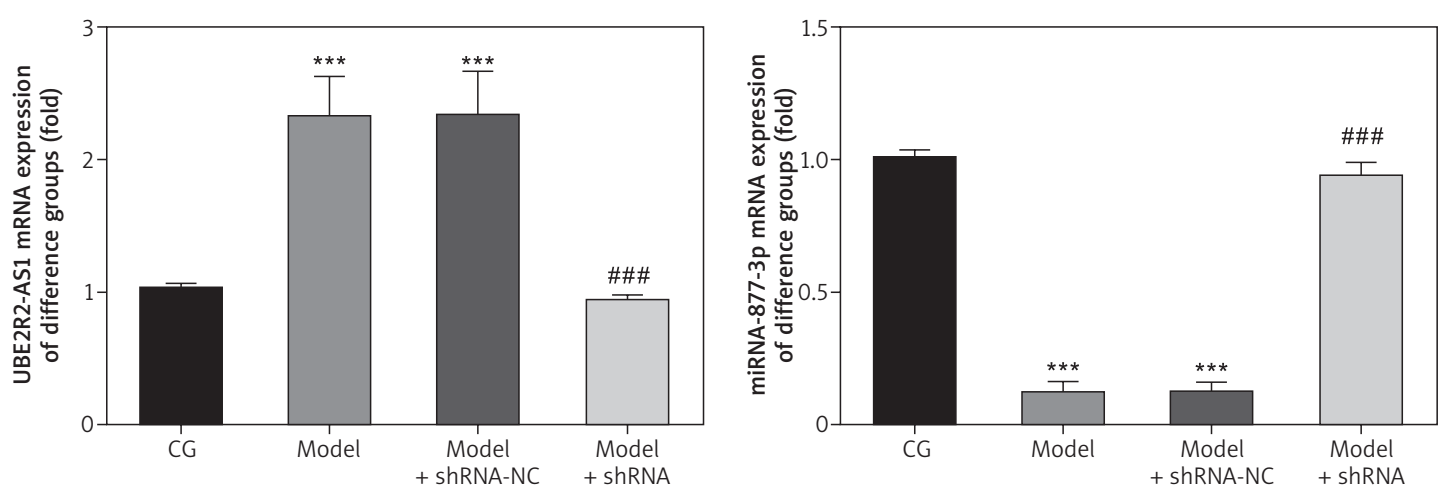

Figure 1. IncRNA UBE2R2-AS1 and miRNA-877-3p gene expression in the different groups CG - control group, Model - high-glucose model group, Model + shRNA-NC - HK-2 cells transfected with shRNA-negative control and treated with high-glucose medium, Model + shRNA - HK-2 cells transfected with ShRNA UBE2R2-AS1 and treated with high-glucose medium. ${ }^{* * *} P<0.001$ vs. NC group; ${ }^{\# \#} p<0.001$ vs. Model group. 
UBE2R2-AS1 mRNA expression was significantly down-regulated $(p<0.001)$ and miRNA-877-3p gene expression was significantly higher $(p<0.001)$ in the model + shRNA group (Figure 1).

\section{Effects of IncRNA UBE2R2-AS1 knockdown on cell apoptosis}

Compared with the NC group, the cell apoptosis rate was significantly higher $(p<0.001)$ in the model and model + shRNA-NC groups (Figure 2). Knockdown of UBE2R2-AS1 using shRNA significantly decreased $(p<0.001)$ the cell apoptosis rate in the model + shRNA group compared with that of the model group (Figure 2).

\section{Effect of IncRNA UBE2R2-AS1 knockdown on inflammatory factors}

ELISA analysis showed that TNF- $\alpha$ and IL- 6 levels were significantly higher in the model and model + shRNA-NC groups compared with those of the CG ( $p<0.001$ for both; Figure 3). Transfection with shRNA UBE2R2-AS1 led to significantly lower TNF- $\alpha$ and IL-6 levels in the model + shRNA group compared with those of the model group $(p<0.001)$ (Figure 3).
CG

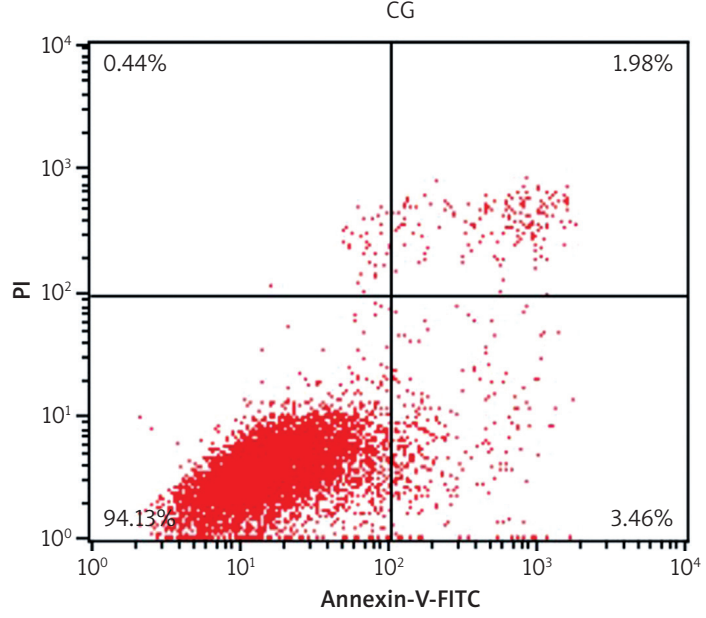

Model + shRNA-NC
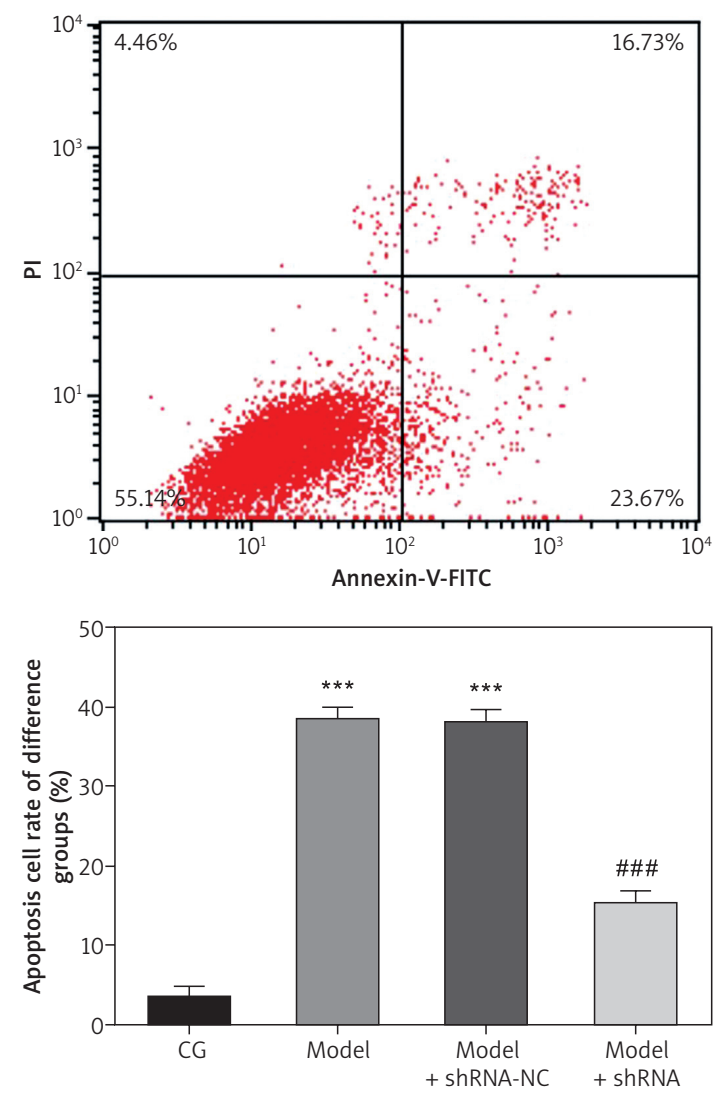

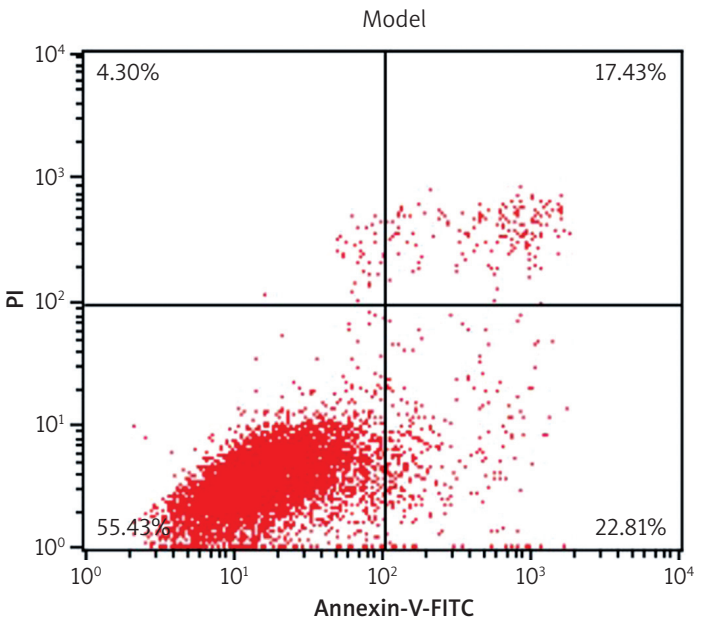

Model + shRNA

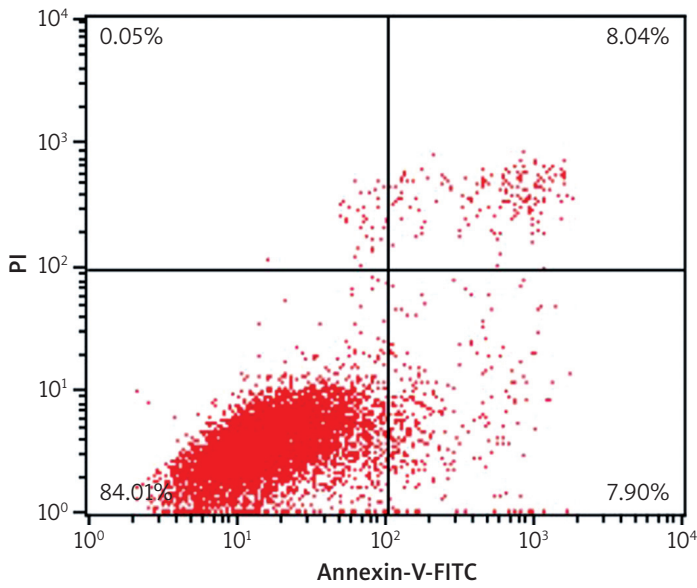

Figure 2. Effects of IncRNA UBE2R2-AS1 knockdown on cell apoptosis

CG - control group, Model - high-glucose model group, Model + shRNA-NC - HK-2 cells transfected with shRNA-negative control and treated with highglucose medium, Model + shRNA - HK-2 cells transfected with shRNA UBE2R2-AS1 and treated with high-glucose medium. ${ }^{* *} P<0.001$ vs. NC group; ${ }^{\# \#} p<0.001$ vs. Model group. 
Effect of IncRNA UBE2R2-AS1 knockdown on relative protein expression measured by WB

Western blot (WB) analysis revealed that TLR4, MyD88 and NF- $\kappa \mathrm{B}(\mathrm{p} 65)$ protein levels were significantly higher $(p<0.001)$ in the model and model
+ shRNA-NC groups compared with those of the CG (Figure 4). Knockdown of IncRNA UBE2R2-AS1 using shRNA led to significantly lower $(p<0.001)$ TLR4, MyD88 and NF- $\kappa$ B(p65) protein levels in the model + shRNA group compared with those of the model group (Figure 4).
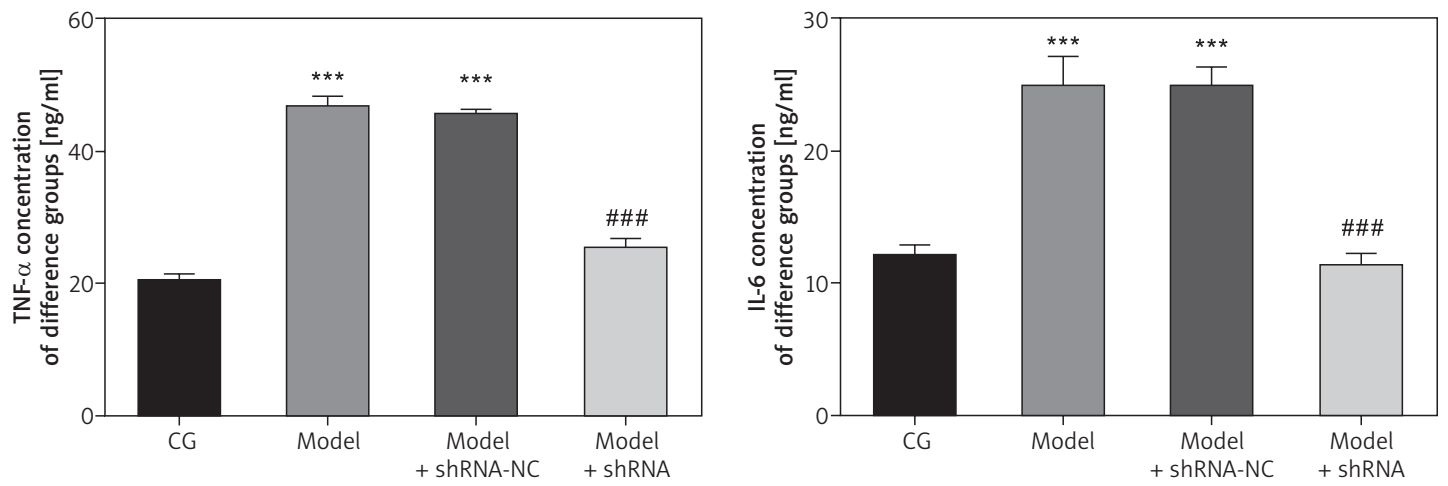

Figure 3. Effect of IncRNA UBE2R2-AS1 knockdown on inflammatory factors

CG - control group, Model - high-glucose model group, Model + shRNA-NC - HK-2 cells transfected with shRNA-negative control and treated with high-glucose medium, Model + shRNA - HK-2 cells transfected with shRNA UBE2R2-AS1 and treated with high-glucose medium. ${ }^{* * *} P<0.001$ vs. NC group; ${ }^{\# \# p}<0.001$ vs. Model group.
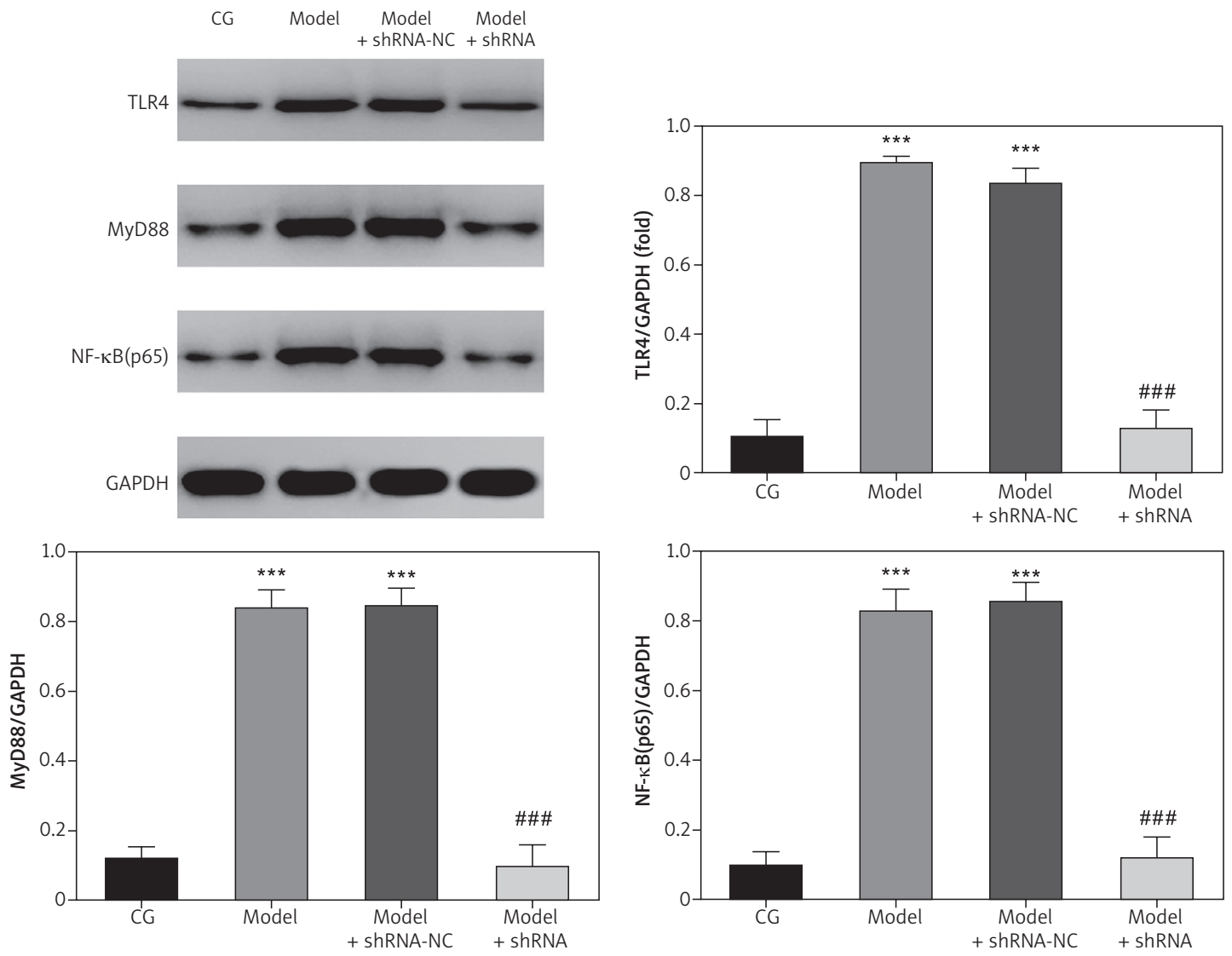

Figure 4. Effects of IncRNA UBE2R2-AS1 knockdown on relative protein expression measured by WB

CG - control group, Model - high-glucose model group, Model + shRNA-NC - HK-2 cells transfected with shRNA-negative control and treated with high-glucose medium, Model + shRNA - HK-2 cells transfected with shRNA UBE2R2-AS1 and treated with high-glucose medium. ${ }^{* *} P<0.001$ vs. NC group; ${ }^{\# \#} p<0.001$ vs. Model group. 
Effect of IncRNA UBE2R2-AS1 knockdown on relative protein expression measured by immunocytochemistry

Immunocytochemistry analysis showed significantly higher TLR4, MyD88 and NF- $\mathrm{KB}(\mathrm{p} 65)$ protein volume $(p<0.001$ for all) in the model and model + shRNA-NC groups compared with the CG (Figure 5). Furthermore, shRNA inhibition of IncRNA UBE2R2-AS1 significantly decreased TLR4, MyD88 and NF- $\kappa B(p 65)$ protein levels in the model + shRNA group $(p<0.001)$ (Figure 5$)$.
mRNA expression of IncRNA UBE2R2-AS1 and miRNA-877-3p in the different groups

Compared with the CG, IncRNA UBE2R2-AS1 mRNA levels were significantly up-regulated ( $p<0.001$ ), whereas miRNA-877-3p mRNA expression was significantly down-regulated $(p<0.001)$ in the model group (Figure 6). shRNA knockdown of IncRNA UBE2R2-AS1 significantly $(p<0.001)$ down-regulated IncRNA UBE2R2-AS1 mRNA expression and miRNA-877-3p mRNA expression was significantly higher $(p<0.001)$ in the model
A
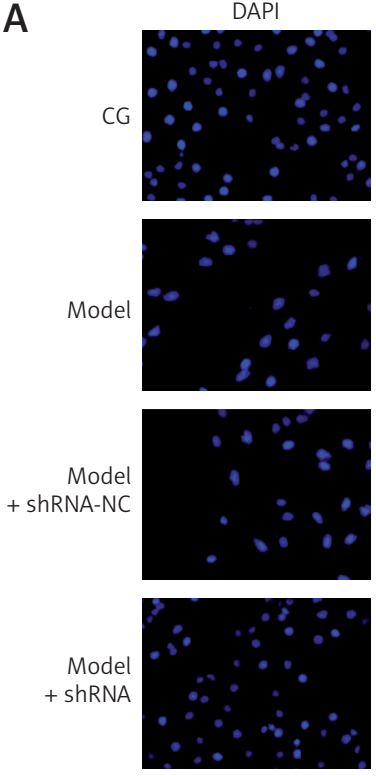

B

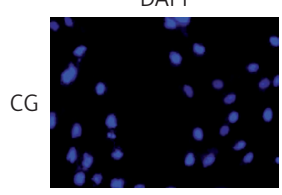

Model
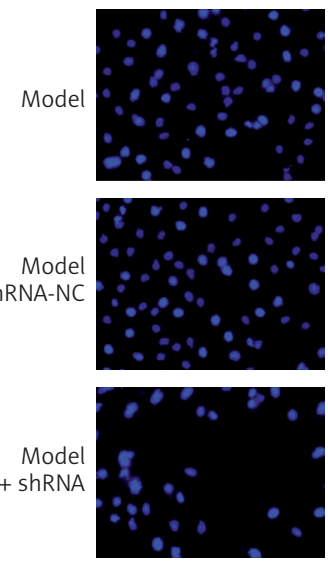

Figure 5. Effects of IncRNA UBE2R2-AS1 knockdown on relative proteins expression measured by immunocytochemistry

CG - control group, Model - high-glucose model group, Model + shRNA-NC - HK-2 cells transfected with shRNA-negative control and treated with high-glucose medium, Model + shRNA - HK-2 cells transfected with shRNA UBE2R2 AS1 and treated with high-glucose medium. ${ }^{* * *} P<0.001$ vs. NC group; ${ }^{\# \#} p<0.001$ vs. Model group.
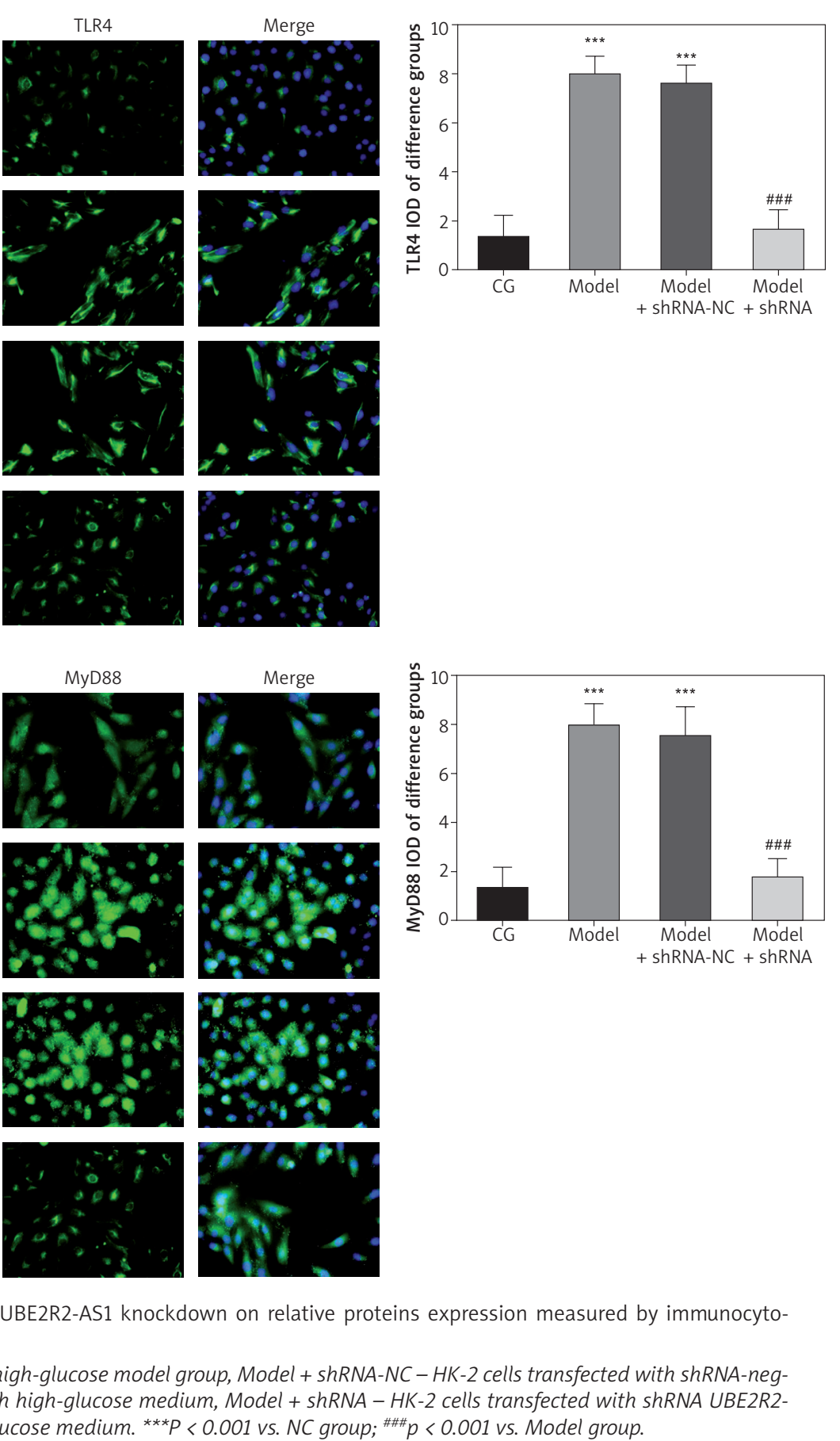
C
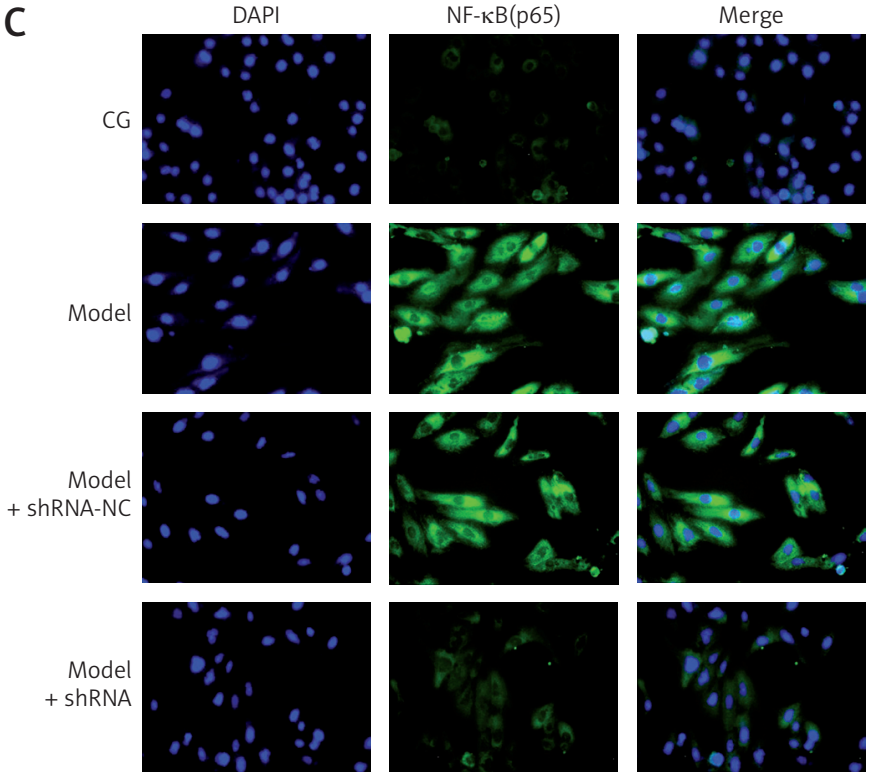

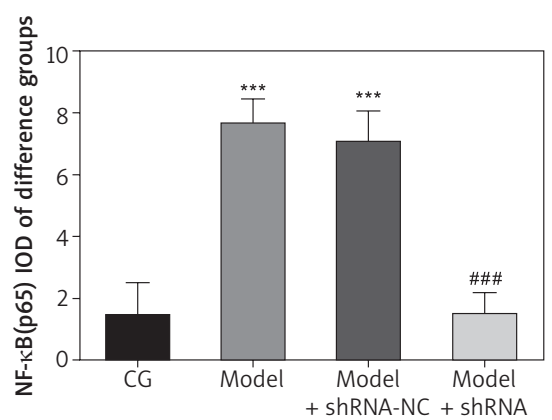

Figure 5. Cont.

CG - control group, Model - high-glucose model group, Model + shRNA-NC - HK-2 cells transfected with shRNA-negative control and treated with high-glucose medium, Model + shRNA - HK-2 cells transfected with shRNA UBE2R2AS1 and treated with high-glucose medium. ${ }^{* * *} P<0.001$ vs. NC group; ${ }^{\# \#} p<0.001$ vs. Model group.
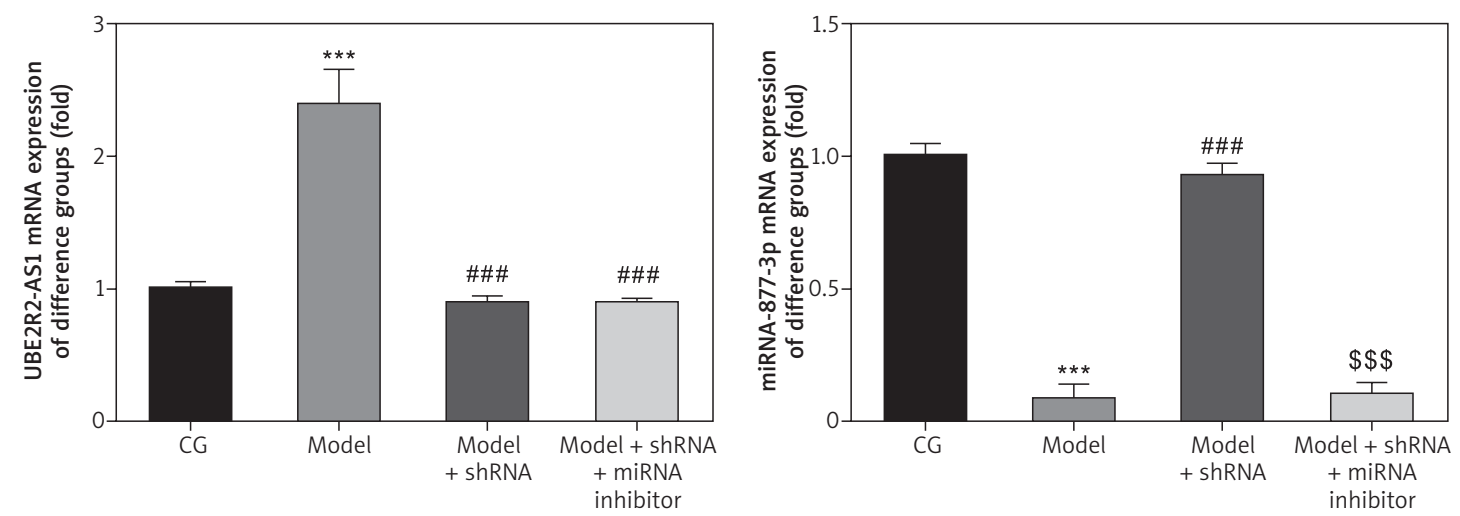

Figure 6. mRNA expression of IncRNA UBE2R2-AS1 and miRNA-877-3p in the different groups

CG - control group, Model - high-glucose model group, Model + shRNA - HK-2 cells transfected with shRNA UBE2R2 AS1 and treated with high-glucose medium, Model + shRNA + miRNA inhibitor - HK-2 cells transfected with shRNA UBE2R2-AS1 and miRNA-877-3p inhibitor and treated with high-glucose medium. ${ }^{* * *} P<0.001$ vs. NC group; ${ }^{\# \#} p<0.001$ vs. Model group, ${ }^{\$ \$ \$} P<0.001$ vs. Model + shRNA group.

+ shRNA group compared with that of the model group (Figure 6). Treatment with shRNA targeting IncRNA UBE2R2-AS1 and miRNA inhibitor resulted in significantly lower miRNA-877-3p and IncRNA UBE2R2-AS1 mRNA expression in the model + shRNA + miRNA inhibitor group $(p<0.001)$ compared with the model + shRNA group.

\section{Flow cytometry analysis of cell apoptosis}

Flow cytometry analysis revealed a significantly higher $(p<0.001)$ cell apoptosis rate in the model group compared with that of the CG, whereas the cell apoptosis rate of the model + shRNA group was significantly lower $(p<0.001)$ compared with that of the model group (Figure 7). The cell apoptosis rate of the model + shRNA + miRNA inhibitor group was significantly higher compared with that of the model + shRNA group $(p<0.001)$ (Figure 7 ).

\section{Measurement of inflammatory factors in the different groups}

ELISA analysis revealed significant up-regulation in TNF- $\alpha$ and IL- 6 levels in the model group compared with those of the CG ( $p<0.001$ for both) (Figure 8). TNF- $\alpha$ and IL-6 levels were significantly down-regulated in the model + shRNA group compared with those of the model group $(p<0.001$ for both). However, levels of these inflammatory 
CG

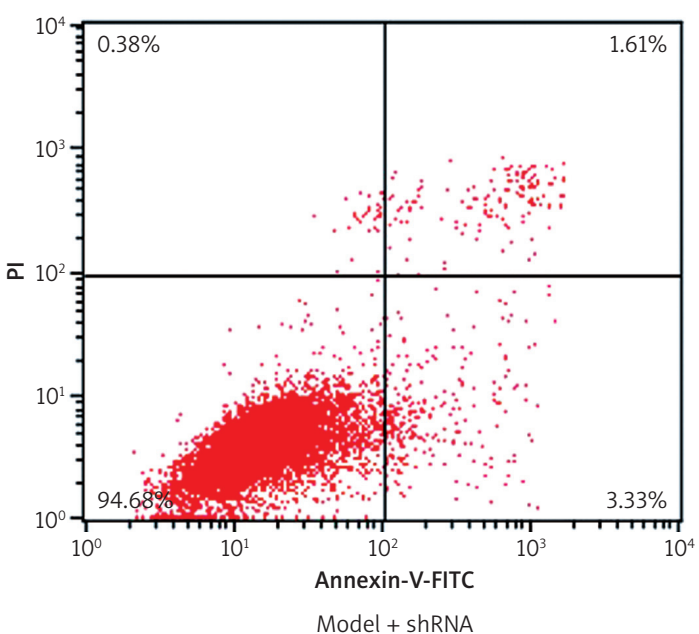

Model + shRNA
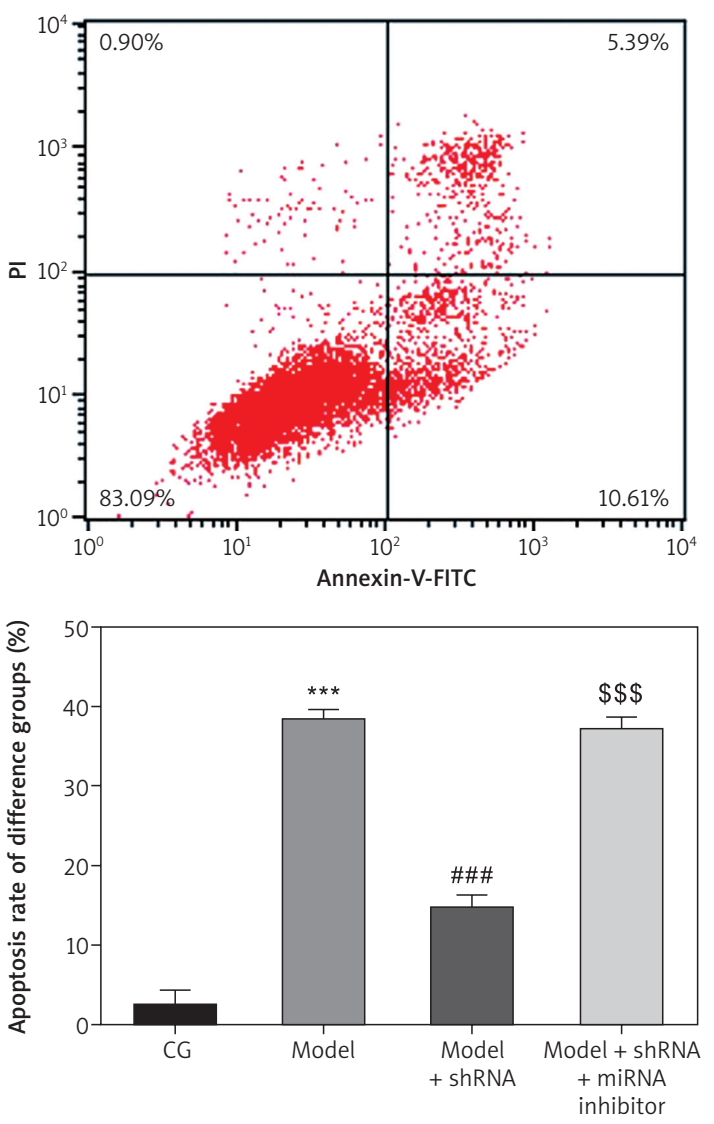

markers were significantly higher $(p<0.001)$ in the model + shRNA + miRNA inhibitor group compared with those of the model + shRNA group (Figure 8).

\section{WB analysis of relative protein levels}

TLR4, MyD88 and NF-kB(p65) proteins levels were significantly up-regulated in the model group compared with those of the CG $(p<0.001$ for all) (Figure 9). Compared with the model group, TLR4, MyD88 and NF-kB(p65) proteins levels were
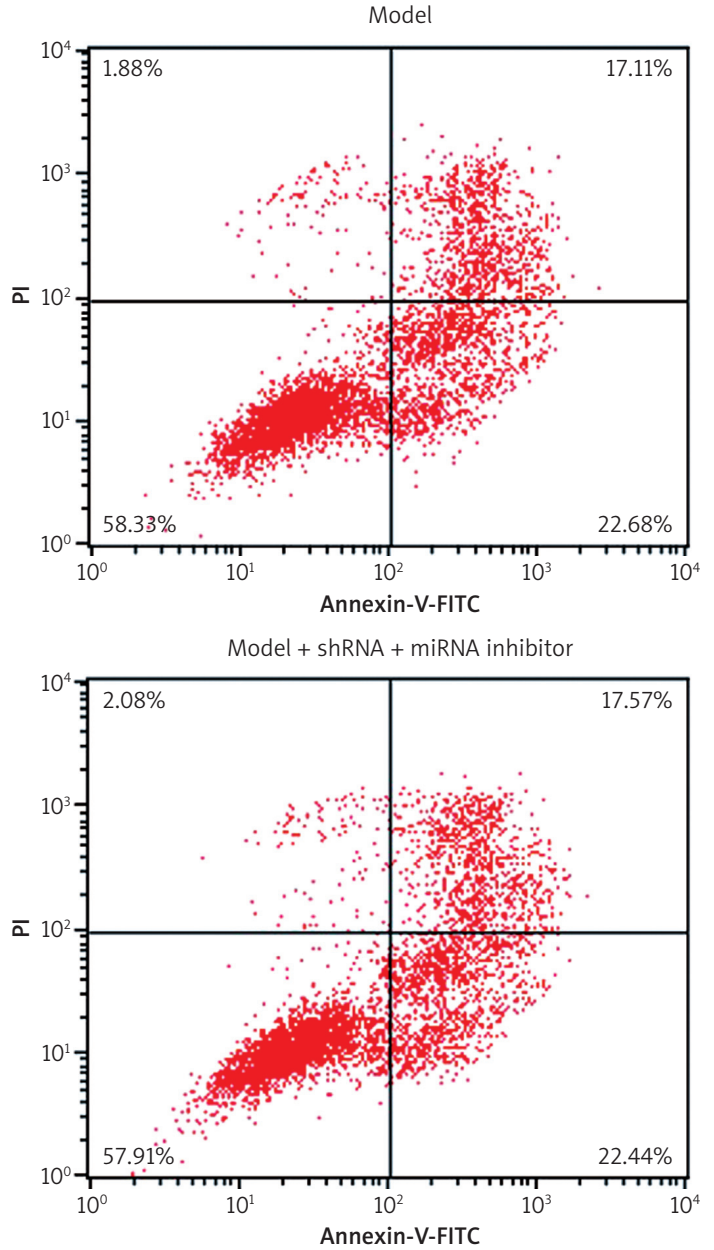

Figure 7. Cell apoptosis in the different groups CG - control group, Model - high-glucose model group, Model + shRNA - HK-2 cells transfected with shRNA UBE2R2-AS1 and treated with high-glucose medium, Model + shRNA + miRNA inhibitor - HK-2 cells transfected with shRNA UBE2R2-AS1 and miRNA-877-3p inhibitor and treated with high-glucose medium. ${ }^{* *} P<0.001$ vs. NC group; ${ }^{* \#} p<0.001$ vs. Model group, ${ }^{S S S} P<0.001$ vs. Model + shRNA group.

significantly down-regulated $(p<0.001)$ in the model + shRNA group (Figure 9). However, the addition of miRNA-877-3p inhibitor significantly increased $(p<0.001)$ expression of these proteins in the model + shRNA + miRNA inhibitor group compared with the model + shRNA group (Figure 9).

\section{Amount of relative proteins among} the different groups

Immunocytochemistry showed significantly higher $(p<0.001)$ amounts of TLR4, MyD88 and 

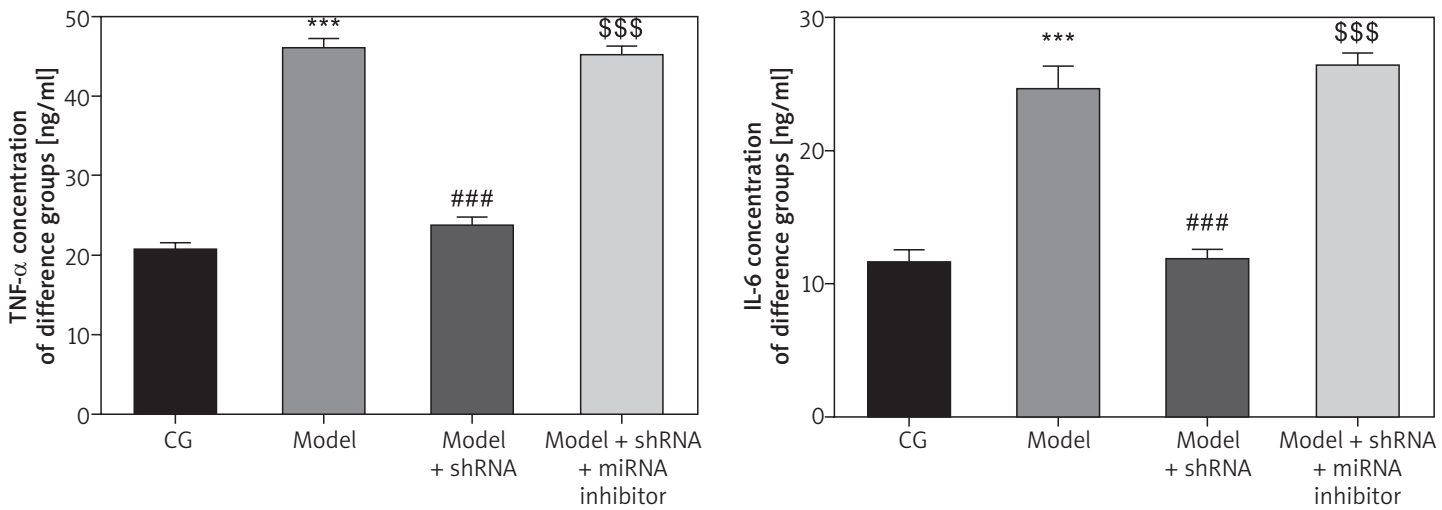

Figure 8. Concentration of inflammatory factors in the different groups measured by ELISA

CG - control group, Model - high-glucose model group, Model + shRNA - HK-2 cells transfected with shRNA UBE2R2AS1 and treated with high-glucose medium, Model + shRNA + miRNA inhibitor - HK-2 cells transfected with shRNA UBE2R2-AS1 and miRNA-877-3p inhibitor and treated with high-glucose medium. ${ }^{* * *} P<0.001$ vs. NC group; ${ }^{\# \# ~} p<0.001$ vs. Model group, $\$ \$ \$ \$ P<0.001$ vs. Model + shRNA group.
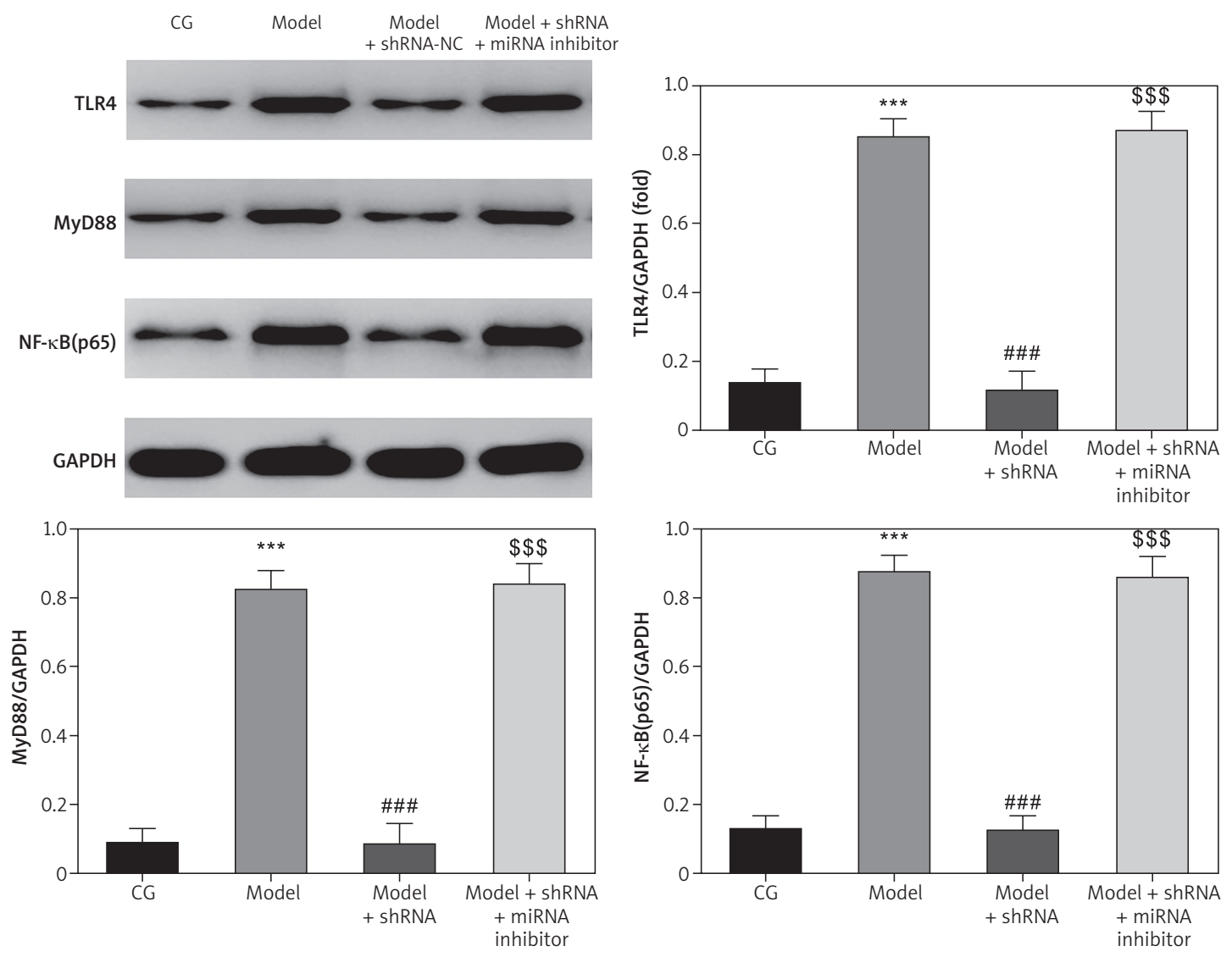

Figure 9. Relative proteins expression measured by WB assay

CG - control group, Model - high-glucose model group, Model + shRNA - HK-2 cells transfected with shRNA UBE2R2AS1 and treated with high-glucose medium, Model + ShRNA + miRNA inhibitor - HK-2 cells transfected with shRNA UBE2R2-AS1 and miRNA-877-3p inhibitor and treated with high-glucose medium. ${ }^{* * *} P<0.001$ vs. NC group; ${ }^{\# \#} p<0.001$ vs. Model group, ${ }^{\$ S \$} P<0.001$ vs. Model + shRNA group.

NF- $\mathrm{K}(\mathrm{p} 65)$ protein in the model group compared with the CG. Amounts of TLR4, MyD88 and NF$\kappa \mathrm{B}(\mathrm{p} 65)$ protein were significantly lower in the model + shRNA group compared with those of the model group ( $p<0.001$ for all). However, addition of miRNA-877-3p inhibitor significantly decreased the amounts of TLR4, MyD88 and NF- $\mathrm{kB}(\mathrm{p} 65)$ protein in the model + shRNA + miRNA inhibitor group compared with those of the model + shRNA group ( $p<0.001$ for all) (Figure 10). 
A
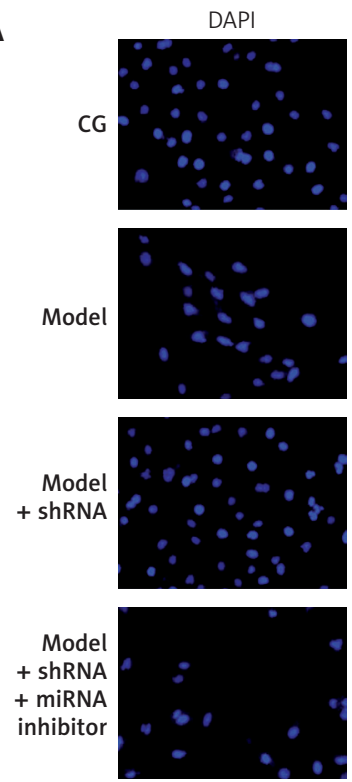

B
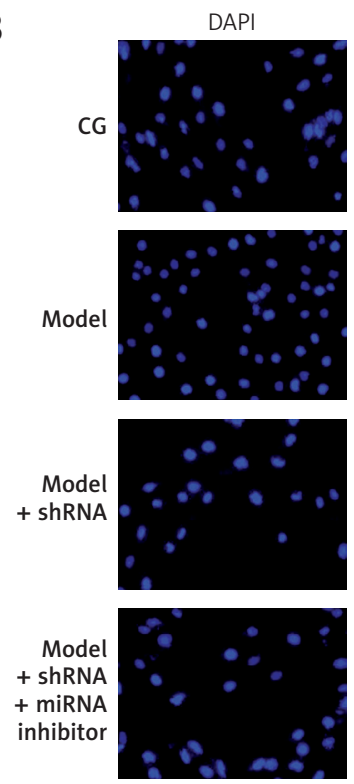

TLR4
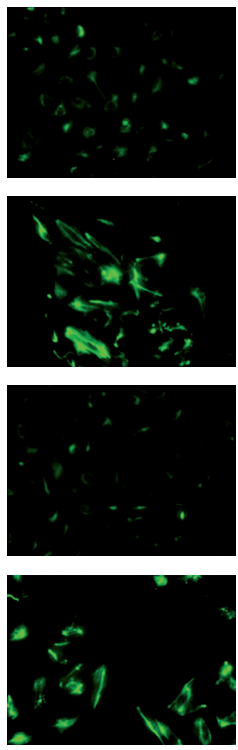

MyD88
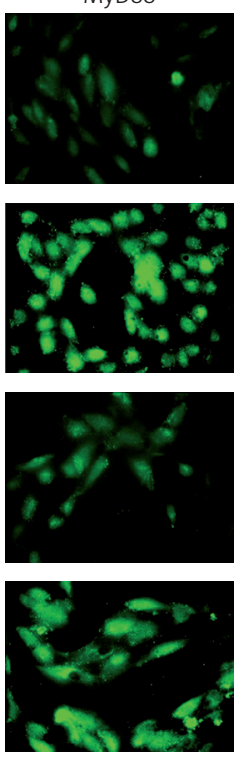

Merge
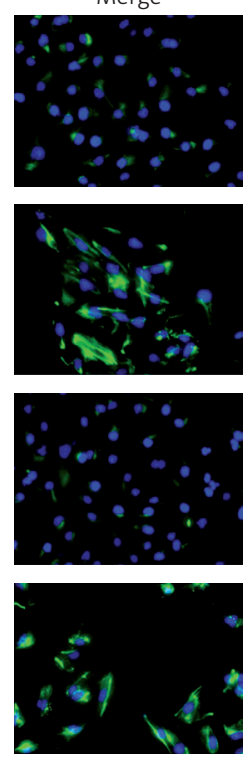

Merge
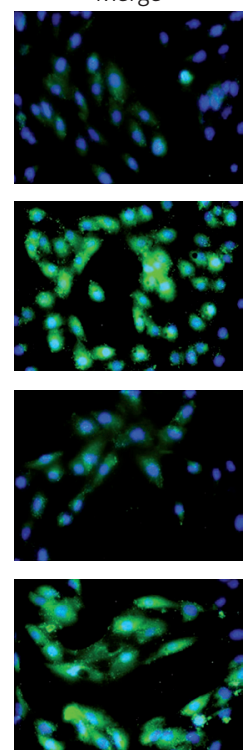

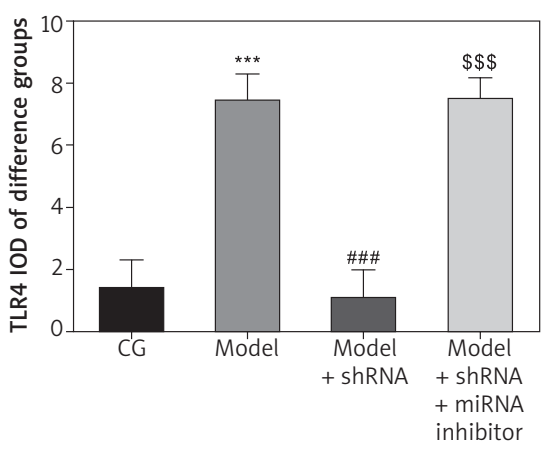

Figure 10. Relative protein amount in the different groups measured by immunocytochemistry

CG - control group, Model - high-glucose model group, Model + shRNA - HK-2 cells transfected with shRNA UBE2R2-

AS1 and treated with high-glucose medium, Model + shRNA + miRNA inhibitor - HK-2 cells transfected with shRNA UBE2R2-AS1 and miRNA-877-3p inhibitor and treated with high-glucose medium. ${ }^{* *} P<0.001$ vS. NC group; ${ }^{\# \# \# p}<0.001$ vs. Model group, ${ }^{\$ \$ \$} p<0.001$ vs. Model + shRNA group.

Correlations between UBE2R2-AS1, miRNA-877-3p and TLR4

Luciferase reporter assay demonstrated that miRNA-877-3p was the target gene of UBE2R2-AS1 in HK-2 cells and that TLR4 was the target gene of miRNA-877-3p in HK-2 cells (Figure 11).

\section{Discussion}

The biological functions of IncRNAs continue to be revealed, including cell cycle control and epigen- etic regulation, as well as roles in transcription, alternative splicing, translation and genetic imprinting. Some IncRNAs have also been detected in urine and blood, enabling their use as biomarkers for in vitro diagnosis. For example, IncRNA PGA3 was detected in urine sediment from prostate cancer patients, and was demonstrated to be more sensitive and specific than prostate-specific antigen as a marker of prostate cancer [11-13]. The IncRNA HOTAIR was detected in the serum of colorectal cancer patients, and was shown to be a poten- 
C
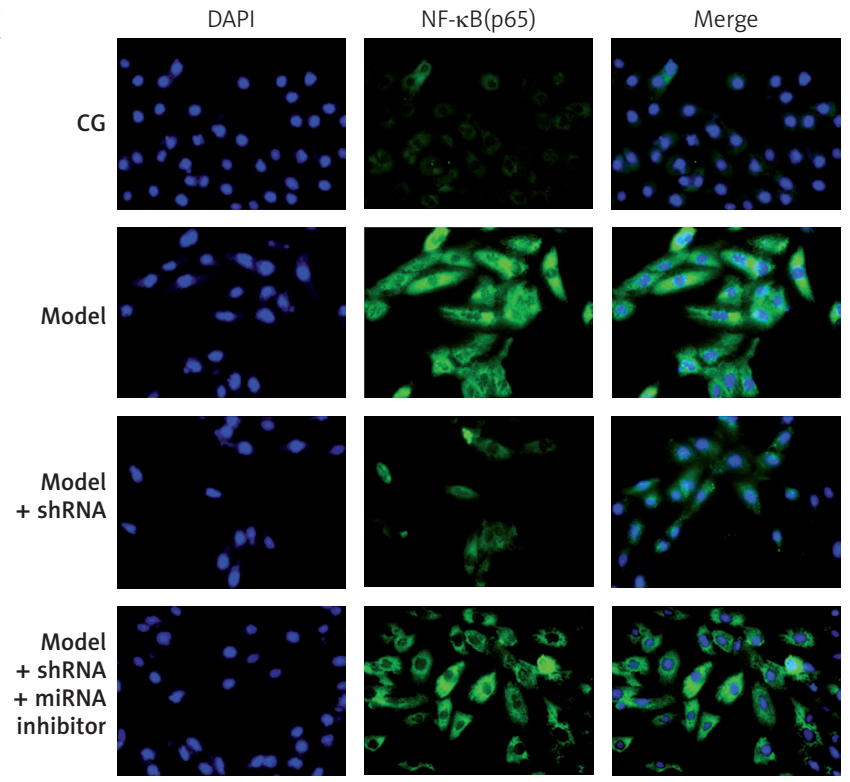

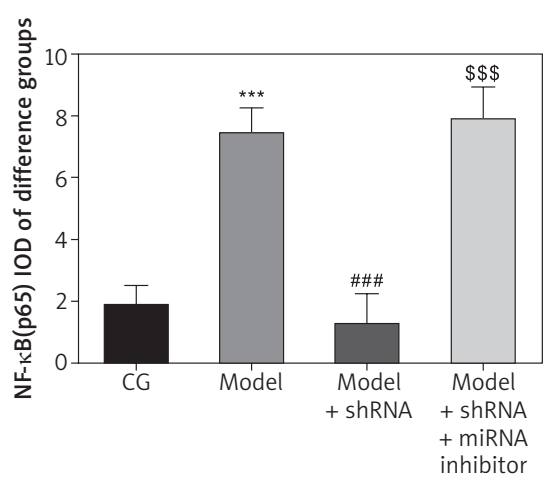

Figure 10. Cont.

CG - control group, Model - high-glucose model group, Model + shRNA - HK-2 cells transfected with shRNA UBE2R2AS1 and treated with high-glucose medium, Model + shRNA + miRNA inhibitor - HK-2 cells transfected with shRNA UBE2R2-AS1 and miRNA-877-3p inhibitor and treated with high-glucose medium. ${ }^{* * *}<0.001 \mathrm{vS.} N C$ group;

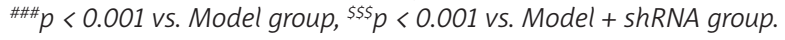

A UBE2R2-AS1-WT 5'-CUGGgaUgGaACAUUGAAGAGGA-3' hsa-miR-877-3p 3'-GACCCUCCUCCCUCUUCUCCU-5, UBE2R2-AS1-MUT 5'-CUGGGAUGGAACAUUCUUCUCCU-3'

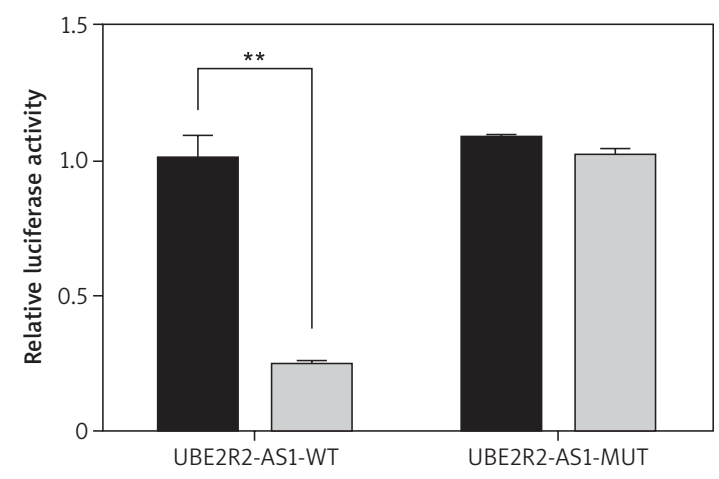

- Mimics-NC $\square$ miR-877-39 mimics

\section{B TLR4 3'UTR-WT 5'-UGGACAUAGAAUAAAAAGAGGA-3' hsa-miR-877-3p 3'-GACCCUCCUCCCUCUUCUCCU-5'

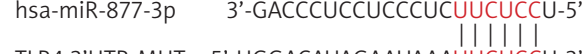

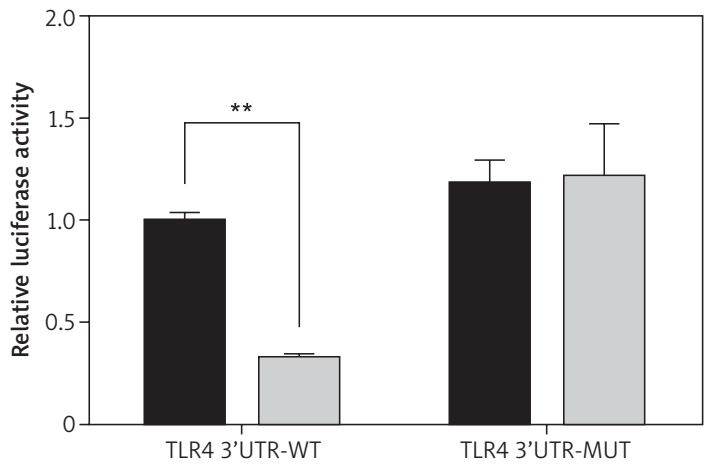

- Mimics-NC $\square$ miR-877-39 mimics

Figure 11. Luciferase reporter assay: A - correlation between UBE2R2-AS1 and miRNA-877-3p in HK-2 cells measured by luciferase reporter assay, B - correlation between miRNA-877-3p and TLR4 in HK-2 cells measured by luciferase reporter assay

${ }^{\star *} P<0.01$ compared with mimics-NC group.

tial biological marker for this disease. In addition, analysis of a small RNA library of plasma exosomes also revealed 3.36\% IncRNA content [14]. Novel techniques, such as RNA immunoprecipitation, sequencing technology, gene chips and RT-PCR have facilitated the clinical application of IncRNA as a non-invasive biomarker. Furthermore, IncRNAs may exist in a stable form in body fluids, suggesting their potential use as novel biomarkers for the clinical diagnosis and prognostic analysis of DKD patients. Related studies have shown that various IncRNAs have regulatory effects on the occurrence and development of DKD [15-20]. In our present study, the results showed that the cell apoptosis was suppressed and inflammation-related factors (TNF- $\alpha$ and IL-6) were improved with IncRNA UBE2R2-AS1 knockdown by shRNA; however, with miRNA-877-3p inhibitor transfection, the cell in- 
jury and TNF- $\alpha$ and IL- 6 concentrations were increased. Based on those results, we inferred that IncRNA UBE2R2-AS1 knockdown had effects to improve diabetes-induced renal cell injury that might be correlated with miRNA-877-3p and the related pathway TLR4/MyD88/NF- $\mathrm{B}(\mathrm{p} 65)$.

Toll-like receptor 4 (TLR4) is the main mediator of immune and inflammatory responses, and its expression in kidney tissue of diabetic kidney injury rats is significantly increased and closely related to proteinuria and renal function [21, 22]. Enhanced expression of TLR4 can increase the activity of NF- $\mathrm{BB}$ via activation of MyD88 activity $[23,24]$. NF- $\kappa B$ is a transcription regulator that widely exists in various tissues and cells and regulates the expression of cytokines and inflammation-mediated genes, and also participates in the progress of various kidney injuries such as diabetic nephropathy [25-27]. The results of the present study suggest that activation of the TLR4/MyD88/ NF- $\kappa B$ pathway was significantly stimulated by cell apoptosis in a high-glucose environment, indicating that activation of this pathway plays a key role in diabetes-induced kidney injury. Our study also revealed that mRNA levels of IncRNA UBE2R2AS1 significantly increased in the model of high glucose-induced renal proximal tubular epithelial cell injury; therefore, UBE2R2-AS1 may play an important role in diabetic kidney injury. Knockdown of UBE2R2-AS1 expression in HK-2 cells using shR$N A$ led to a significant decrease in high-glucoseinduced apoptosis, significant down-regulation of inflammatory factors TNF- $\alpha$ and IL-6, and significant inhibition of the TLR4/MyD88/NF- $\kappa B$ signalling pathway. These results suggest that IncRNA UBE2R2-AS1 can inhibit secretion of inflammatory cytokines by inhibiting the TLR4/MyD88/NF- $\kappa B$ signalling pathway, thus alleviating high-glucoseinduced renal cell injury.

In conclusion, studies have shown that IncRNAs plays a role in the regulation of miRNAs. Among these, IncRNA Tu1 downregulates expression of miR-377, alleviates its inhibition of the target gene PPAR, and reduces ECM accumulation of mesangial cells [28]. Meanwhile, studies have also suggested that IncRNA MALAT1 can alleviate apoptosis in DKD by regulating miR-23c expression [29]. It was previously reported [12] that UBE2R2-AS1 can target miR-877-3p in glioma cells. In the present study, the Dual Luciferase assay confirmed that miR-877-3p is also a target of UBE2R2-AS1 in HK-2 cells, and that miR-877-3p can specifically target TLR4. In conclusion, the present study demonstrates that IncRNA UBE2R2-AS1 improves diabetic kidney injury by targeting miRNA-877-3p/TLR 4.

\section{Conflict of interest}

The authors declare no conflict of interest.

\section{References}

1. IDF. Diabetes atlas-Home. 2016.

2. Yu R, Zhang Y, Lu Z, et al. Long-chain non-coding RNA UCA1 inhibits renal tubular epithelial cell apoptosis by targeting microRNA-206 in diabetic nephropathy. Arch Physiol Biochem 2019; 12: 1-9.

3. Kato $M$, Wang $M$, Chen Z, et al. An endoplasmic reticulum stress regulated IncRNA hosting a microRNA megacluster induces early features of diabetic nephropathy. Nat Commun 2016; 7: 12864.

4. Kaczmarczyk-Sedlak I, Folwarczna J, Sedlak L, et al. Effect of caffeine on biomarkers of oxidative stress in lenses of rats with streptozotocin-induced diabetes. Arch Med Sci 2019; 15: 1073-80.

5. Guo J, Liu Z, Gong R. Long noncoding RNA: an emerging player in diabetes and diabetic kidney disease. Clin Sci 2019; 133: 1321-39.

6. Santer L, López B, Ravassa S, et al. Circulating long noncoding RNA LIPCAR predicts heart failure outcomes in patients without chronic kidney disease. Hypertension 2019; 73: 820-8.

7. Wang M, Wang S, Yao D, et al. A novel long non-coding RNA CYP4B1-PS1-001 regulates proliferation and fibrosis in diabetic nephropathy. Mol Cell Endocrinol 2016; 426: 136-45.

8. Klisic A, Kavaric N, Stanisic V, et al. Endocan and a novel score for dyslipidemia, oxidative stress and inflammation (DOI score) are independently correlated with glycated hemoglobin $(\mathrm{HbA} 1 \mathrm{c})$ in patients with prediabetes and type 2 diabetes. Arch Med Sci 2019; 16: 42-50.

9. Zhu L, Zhao S, Liu S, et al. PTEN regulates renal extracellular matrix deposit via increased CTGF in diabetes mellitus. J Cell Biochem 2016; 117: 1187-98.

10. McClelland AD, Herman-Edelstein M, Komers R, et al. miR-21 promotes renal fibrosis in diabetic nephropathy by targeting PTEN and SMAD7. Clin Sci 2015; 129: 1237-49.

11. Zhou M, Zhang Z, Zhao $\mathrm{H}$, et al. An immune-related six-IncRNA signature to improve prognosis prediction of glioblastoma multiforme. Mol Neurobiol 2018; 55: 3684-97.

12. Xu W, Hu GQ, Da Costa C, et al. Long noncoding RNA UBE2R2-AS1 promotes glioma cell apoptosis via targeting the miR-877-3p/TLR4 axis. Onco Targets Ther 2019; 12: 3467-80.

13. Verhoeven RJA, Tong S, Mok BW, et al. Epstein-Barr virus BART long non-coding RNAs function as epigenetic modulators in nasopharyngeal carcinoma. Front Oncol 2019; 9: 1120.

14. Luo J, Qu J, Wu DK, et al. Long non-coding RNAs: a rising biotarget in colorectal cancer. Oncotarget 2017; 8: 22187-202.

15. Dey N, Bera A, Das F, et al. High glucose enhances microRNA-26a to activate mTORC1 for mesangial cell hypertrophy and matrix protein expression. Cell Signal 2015; 27: 1276-85.

16. Zhou L, Xu DY, Sha WG, Shen L, Lu GY, Yin X. Long noncoding MIAT mediates high glucose-induced renal tubular epithelial injury. Biochem Biophys Res Commun 2015; 468: 726-32.

17. Yan B, Yao J, Liu JY, et al. IncRNA-MIAT regulates microvascular dysfunction by functioning as a competing endogenous RNA. Circ Res 2015; 116: 1143-56.

18. Liao J, He Q, Li M, et al. LncRNA MIAT: Myocardial infarction associated and more. Gene 2016; 578: 158-61.

19. Li Z, Shen J, Chan MT, et al. TUG1: a pivotal onvogeic long non-coding RAN of human cancers. Cell Prolif 2016; 49: 471-5. 
20. Li J, Zhang M, An G, et al. LncRNA TUG1 acts as a tumor suppressor in human glioma by promoting cell apoptosis. Exp Biol Med 2016; 241: 644-9.

21. Gong DJ, Wang L, Yang YY, et al. Diabetes aggravates renal ischemia and reperfusion injury in rats by exacerbating oxidative stress, inflammation, and apoptosis. Ren Fail 2019; 41: 750-61.

22. Zhu L, Han J, Yuan R, et al. Berberine ameliorates diabetic nephropathy by inhibiting TLR4/NF-kappaB pathway. Biol Res 2018; 51: 9.

23. Su S, Zhang P, Zhang Q, Yin Z. GSK-3beta Inhibitor Induces expression of the TLR4/MyD88/NF-kappaB signaling pathway to protect against renal ischemia-reperfusion injury during rat kidney transplantation. Inflammation 2019; 42: 2105-18.

24. Lei J, Fu Y, Zhuang Y, et al. miR-382-3p suppressed IL-1beta induced inflammatory response of chondrocytes via the TLR4/MyD88/NF-kappaB signaling pathway by directly targeting CX43. J Cell Physiol 2019; 234: 23160-8.

25. Ibrahim YF, Moussa RA, Bayoumi AMA, et al. Tocilizumab attenuates acute lung and kidney injuries and improves survival in a rat model of sepsis via down-regulation of NF-kappaB/JNK: a possible role of P-glycoprotein. Inflammopharmacology 2020; 28: 215-30.

26. Yang ZJ, Wang HR, Wang YI, et al. Myricetin attenuated diabetes-associated kidney injuries and dysfunction via regulating nuclear factor (erythroid derived 2)-like 2 and nuclear factor-kappaB signaling. Front Pharmacol 2019; 10: 647.

27. Ma F, Li L, Wang Q, et al. Qi-dan-di-huang decoction alleviates diabetic nephropathy by inhibiting the NF-kappaB pathway. Front Biosci 2019; 24: 1477-86.

28. Duan LJ, Ding M, Hou LJ, et al. Long noncoding RNA TUG1 alleviates extracellular matrix accumulation via mediating microRNA-377 targeting of PPARgamma in diabetic nephropathy. Biochem Biophys Res Commun 2017; 484: 598-604.

29. Li X, Zeng L, Cao C, et al. Long noncoding RNA MALAT1 regulates renal tubular epithelial pyroptosis by modulated miR-23c targeting of ELAVL1 in diabetic nephropathy. Exp Cell Res 2017; 350: 327-35. 